\title{
Unveiling excited-state chirality of Binaphthols by sub-picosecond circular dichroism and quantum-chemical calculations
}

\author{
P. Changenet and F. Hache \\ Laboratoire d'Optique et Biosciences, CNRS, INSERM, Ecole polytechnique, IP Paris, \\ 91128 Palaiseau cedex, France \\ francois.hache@polytechnique.edu
}

\begin{abstract}
Time-resolved circular dichroism (TR-CD) is a powerful tool for probing the conformational dynamics of molecules over large time-scales. By using Binaphthol and two bridged derivatives (PL1 and PL2) as chiral prototypes, we present the first comprehensive study of this type in the middle UV, combining femtosecond TR-CD and quantum mechanical calculations (TD-DFT). We show that excitation of the three compounds induces large variations of their transient CD signals, in sharp contrast to those of their achiral transient absorption, which arise both from the alteration of the electronic distribution and the change of the dihedral angle in the excited state.
\end{abstract}

Keywords: Time-resolved circular dichroism, Binaphthol derivative, quantum calculations

\section{INTRODUCTION}

Chiral molecules have a great importance in chemical as well as biochemical processes. They are characterized by the existence of two mirror-imaged enantiomers that have similar properties but interact differently with chiral objects. As far as interaction with light is concerned, this corresponds to chiroptical activity among which the circular dichroism (CD) which is the difference in absorption for a left or a right polarized light. ${ }^{1}$ Molecular chirality can arise from assymmetric centers, but actually, in many pertinent cases, it arises from a chiral arrangement of achiral components. This chirality has a clear geometrical origin and can be found in many chemical compounds and in the majority of biomolecules such as proteins or DNA. ${ }^{2}$ Changes in the conformation of such molecules play a prominent role in the chemical reactivity of such compounds, but they are usually not easy to monitor. We thus propose to use the fact that a change of conformation in those molecules induces a change in the CD of the molecules to gain information on the dynamics of photoexcited chiral molecules by developing a time-resolved CD experiment capable of monitoring the change of CD on ultrashort timescales.

Among the chemical molecules whose chirality comes from the geometrical arrangement, binaphthols (BINOL) are very famous examples. They are composed of two naphthol moieties with an angle (the dihedral angle) between them which provides the asymmetry responsible for the chirality. ${ }^{3}$ This article presents experimental results obtained on the dynamics of the dihedral angle in BINOL after photoexcitation. A previous study had been released, ${ }^{4}$ but the present study strongly complement the previous ones in two aspects. First, two other bridged derivatives of BINOL are studied, emphasizing the importance of the dangling $\mathrm{OH}$ groups in BINOL. Second, a thorough TD-DFT calculation is carried out to give relevant information on the geometry of these molecules in the ground state as well as in the excited state and the consequences in terms of absorption and CD.

\section{MATERIALS AND METHODS}

\subsection{Samples}

We have studied three samples which are depicted in figure 1. BINOL samples were directly purchase from SigmaAldrich with a purity $>97 \%$ whereas the two bridged samples, PL1 and PL2, were synthesized as explained in Ref. [5]. These samples are composed of two moieties which can be arranged in a cisoid or transoid form depending on the value of the dihedral angles. The molecules were dissolved in various solvents as explained below, with a concentration of a few $10^{-4}$ mol. $\mathrm{l}^{-1}$ and placed in $1 \mathrm{~mm}$-thick silica cell. Steady-state absorption and CD were recorded with a double-beam Cary 100 spectrophotometer and a homemade spectro-polarimeter, respectively. 
Figure 2 reports the absorption and CD spectra of the three samples dissolved in cyclohexane. The absorption spectra display three peaks attributed to the ${ }^{1} \mathrm{~L}_{\mathrm{b}},{ }^{1} \mathrm{~L}_{\mathrm{a}}$ and ${ }^{1} \mathrm{~B}_{\mathrm{b}}$ electronic states. In the presence of bridges, one can observe a shift of the various bands related to the steric hindrance brought by the bridges. The CD spectra exhibit the characteristic excitonic bisignate structure in the UV due to the coupling between the ${ }^{1} \mathrm{~B}_{\mathrm{b}}$ transitions of the two moieties

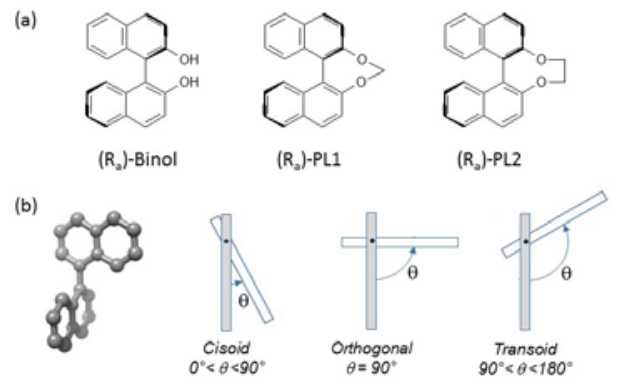

Figure 1 Schematic representations of (a) the three studied 2,2'-homosubstituted 1,1'-binaphthyl compounds: (Ra)-binol, (Ra)-PL1 and (Ra)-PL2, (b) 1,1'-binaphthyl conformers.

\subsection{Time-resolved circular dichroism}

In order to monitor the CD of BINOL and derivatives, two challenges must be overcome. On the one hand, one needs to obtain sub-picosecond pulses tunable in the relevant frequency range, around $230 \mathrm{~nm}$ and on the other hand, one must find a way to measure the CD. The starting point is an amplified Titanium-sapphire laser delivering $3 \mathrm{~mJ}$ pulses at 800 $\mathrm{nm}$. To access the UV range, we first generate a white-light continuum in a sapphire plate which we amplify by an OPA pumped by the second harmonic of the laser beam. This yields an intense pulse tunable in the visible which we frequency-double in a BBO crystal. The final pulse in tunable between 230 and $300 \mathrm{~nm}$, with a duration of about 700 fs and an energy of 150nJ.

The experimental set-up is based on a classical pump-probe geometry. We send onto the sample a $0.5 \mu \mathrm{J}, 267 \mathrm{~nm}$ beam (the third harmonic of the fundamental laser) which can excite the BINOL electronic states, and we probe the excitedstate CD and its time evolution with a delayed probe pulse at $234 \mathrm{~nm}$, which corresponds to the largest amplitude of the static CD signal for all samples. In order to measure the CD, we implement the technique we have proposed in ref [6]: the sample is placed between two crossed polarizers and a Babinet-Soleil compensator is inserted after the sample. By tuning the retardation of the Babinet-Soleil compensator and measuring the intensity transmitted through the crossed analyzer, we can measure the pump-induced CD change as a function of the pump-probe delay and reconstruct the dynamics of the excited-state CD.

\subsection{TD-DFT}

The electronic structure of BINOL and derivatives is quite complex since at least three transitions are involved: ${ }^{1} \mathrm{~L}_{\mathrm{a}},{ }^{1} \mathrm{~L}_{\mathrm{b}}$ and ${ }^{1} \mathrm{~B}_{\mathrm{b}}$. In order to gain a more precise view of the interplay between the electronic structure and the experimental measurements, we have developed TD-DFT calculations using the M052X and CAM-B3LYP functionals. Geometry optimization was performed by using the 6-31G(d) basis set, whereas the energies were refined by using the larger 6$31+G(d, p)$ basis set. Solvent (cyclohexane) effects were included in the calculations implicitly by the Polarizable Continuum Model (PCM). All the calculations were performed using the Gaussian 09. We also adopted DALTON2016 to compute the excited-state absorption and CD from the lowest four excited states and the transition moments between excited states from the double residues of quadratic response functions. Due to their computational cost, calculations were only performed in gas-phase with the small 6-31G(d) basis set. More details can be found in Ref. [7]. 

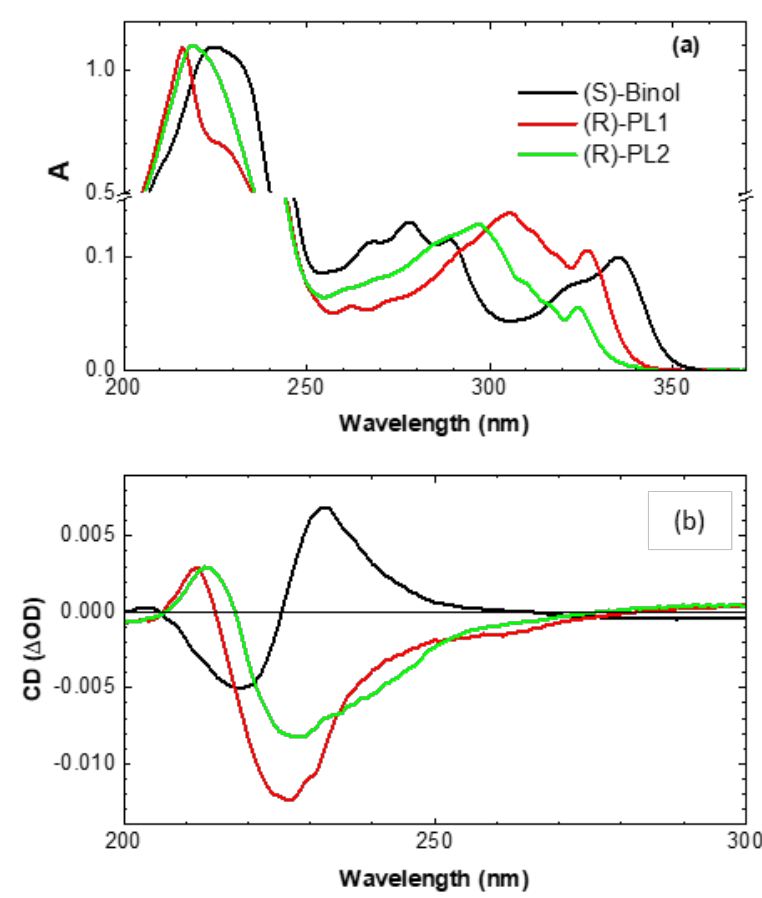

Figure 2. (a) Static absorption and (b) CD spectra of (S)-BINOL (black lines), (R)-PL1 (red lines) and (R)-PL2 (green lines) in Ethanol.

\section{TIME-RESOLVED CD}

\subsection{Experiments}

A full set of experiments have been carried out for the three samples. We have first checked that the transient signals have opposite signs for R and S enantiomers (not shown), as expected for a chiral signal. In all cases, we observe an instantaneous decrease (in absolute value) of the $\mathrm{CD}$ which is attributed to the bleaching of the ground state CD followed by a decrease of the excited state CD signal. Since this dynamics is not visible in transient absorption signals, it is clearly connected to changes in the molecular conformation. The interesting feature worth pointing out is the variety of response times depending on the molecule under study and on the solvents. Three solvents were utilized : Ethanol, Cyclohexane and Ethylene Glycol ; the results are summarized in figure 3. The main points of interest are now described :

- for all samples, one observes a strong decrease of the dynamics when one uses Ethylene Glycol. This is clearly related to the much strongest viscosity of this solvent compared to the other two, which indicates that there is a real change of the dihedral angle in the excited molecules implying a motion of the moieties that is slowed down because of viscosity.

- considering the responses in Ethanol and Cyclohexane which have similar viscosities, one can see that BINOL and PL1/2 do not behave the same. Whereas in PL1/2, both solvents yield identical dynamics, it is not the case in BINOL where the response is more rapid in Cyclohexane. This feature can be traced back to the presence of pending OH groups in BINOL, absent in PL1/2. The slowing down of the response of BINOL in Ethanol is therefore a consequence of Hydrogen-bonds between the $\mathrm{OH}$ groups and the solvent.

- interestingly, one can observe that the dynamics in Ethylene Glycol is more rapid in PL1 and PL2 than in BINOL. This intriguing feature is counterintuitive since motion of the moieties is expected to be easier in the non-bridged BINOL. This point will be discussed in the next section. 

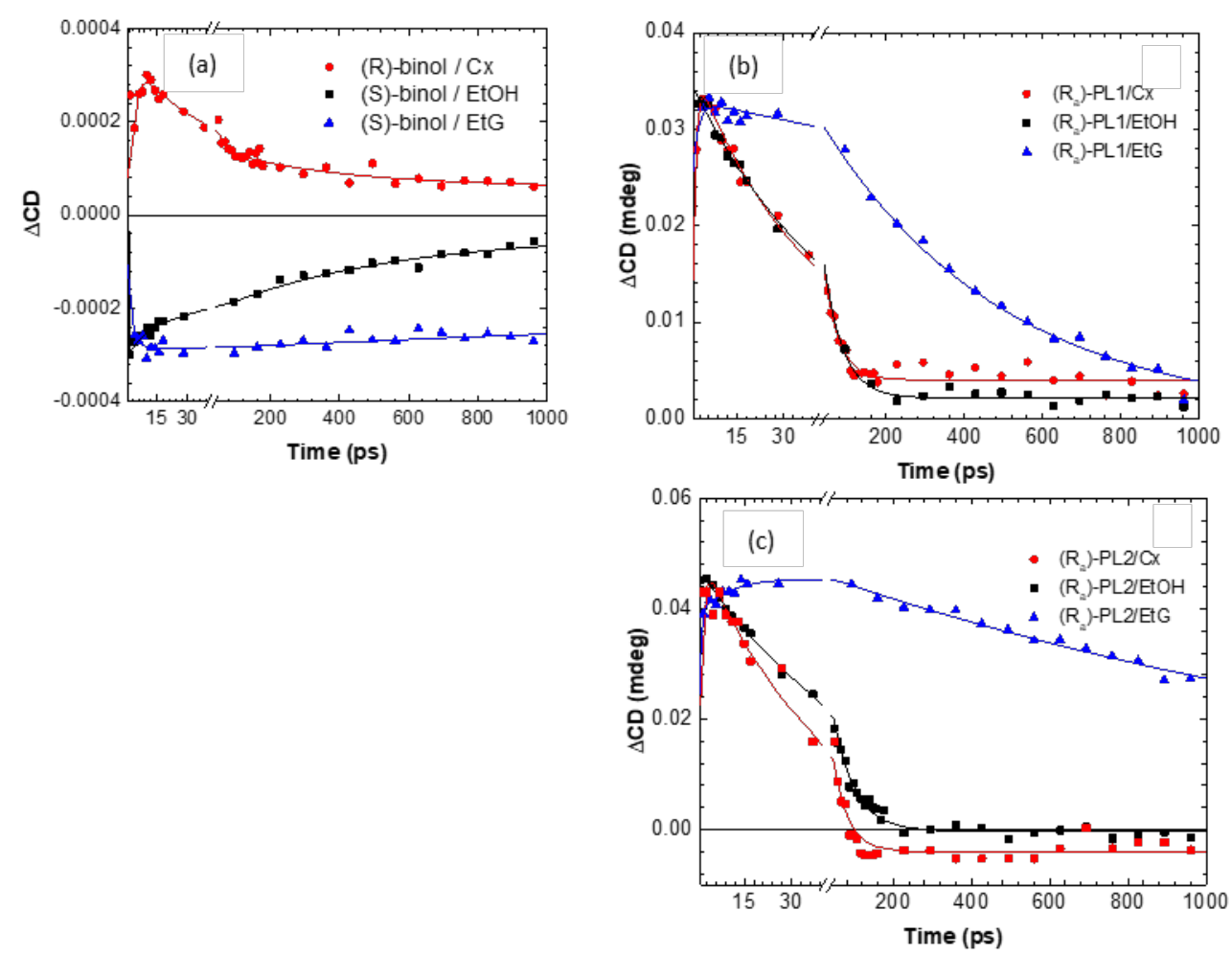

Figure 3. Comparison of the normalized TR-CD changes measured after 1ps at $234 \mathrm{~nm}$ in Ethanol (EtOH), Cyclohexane (Cx) and Ethylene Glycol (EtG), for (a) BINOL, (b)PL1, (c) PL2. Solid lines represent the individual fits of the experimental data. Excitation: $266 \mathrm{~nm}$.

\subsection{Theoretical calculations}

Geometry optimization in the ground state shows that BINOL is very flexible and can have a broad range of conformations from cisoid to transoid ones $\left(70^{\circ}<\theta<110^{\circ}\right)$. On the contrary, the bridges reduce the flexibility of the dihedral angle of PL1 and PL2 and they exhibit ony a cisoid conformation with their dihedral angle equal to $49^{\circ}$ and $61^{\circ}$ respectively. As a result, the positions of the ${ }^{1} \mathrm{~L}_{\mathrm{a}}$ and ${ }^{1} \mathrm{~L}_{\mathrm{b}}$ bands get closer in the bridged samples, resulting in the merging of the two lowest transitions as observed in figure 2. Figure 4 displays the calculated CD spectrum of BINOL for various levels of theory ${ }^{7}$. The overall excitonic shape is well recovered by the calculation.

Thanks to TD-DFT, it was possible to access information on the excited-state geometry and indeed, it was observed that the dihedral angle in BINOL can change towards a more planar geometry, consistent with the observation of a viscositydependent relaxation time. Another interesting feature is the inversion of the ${ }^{1} \mathrm{~L}_{a}$ and ${ }^{1} \mathrm{~L}_{b}$ transitions when the dihedral angle changes, inducing population transfer between the two states after photoexcitation. This feature explains the difference between BINOL and PL1/2 in Ethylene Glycol. Indeed, the population transfer is more efficient in the bridged samples that have a marked cisoid conformation, whereas, in BINOL where the dihedral angle is closer from $90^{\circ}$, it is less efficient. The observed difference in the CD dynamics in Ethylene Glycol therefore shows that the change in the excited state $\mathrm{CD}$ is an interplay between the geometric change (closing of the dihedral angle) and the electronic reorganization (inversion of ${ }^{1} \mathrm{~L}_{\mathrm{a}}$ and ${ }^{1} \mathrm{~L}_{\mathrm{b}}$ ). 


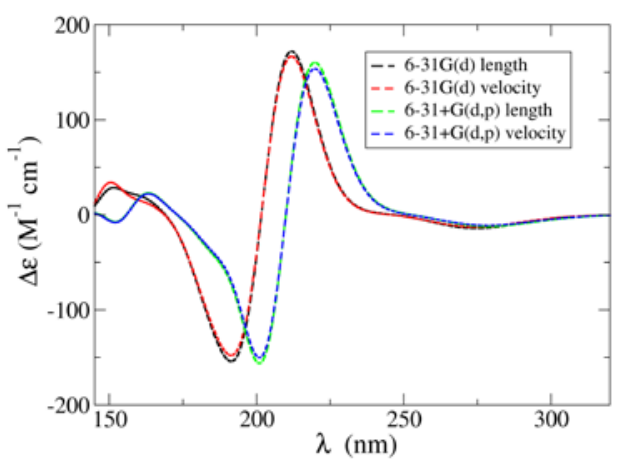

Figure 4 Comparison of the calculated CD $\mathrm{GS}_{\text {s }}$ spectra of $\left(\mathrm{S}_{\mathrm{a}}\right)$-BINOL in gas phase obtained at CAM-B3LYP/6-31G(d) level of theory with length and velocity gauges.

\section{CONCLUSION}

In conclusion, we presented the first comprehensive sub-picosecond TR-CD study of BINOL and two bridged derivatives and examined the influence of the solvents. Upon excitation, we observed significant changes in the TR-CD signals caused by the chirality change of the excited state. Combination of TR-CD and quantum chemical calculations brought evidence that those effects stem from the intricate changes of the electronic structure and the conformation of biaryls upon excitation.

\section{ACKNOWLEDGMENTS}

We thank Lara Martinez-Fernandez, Fabrizio Santoro and Roberto Improta (University of Pisa, Italy) for the quantum calculations and numerous discussions.

We thank Laure Guy (ENS-Lyon, France) and Stéfan Guy (ILM-Lyon, France) for providing us with the PL1 and PL2 samples.

\section{REFERENCES}

[1] Rodger, A.; Norden, B. Circular dichroism and linear dichroism; Oxford University Press, Oxford, UK (1997).

[2] Fasman, G. D. Circular Dichroism and the conformational analysis of biomolecules. Plenum Press, New York (1996).

[3] Bari, L. D.; Pescitelli, G.; Salvadori, P., "Conformational Study of 2,2'-Homosubstituted 1,1'-Binaphthyls by Means of UV and CD Spectroscopy", J. Am. Chem. Soc. 121, 7998-8004 (1999).

[4] Niezborala, C.; Hache, F., "Conformational changes in photoexcited (R)-(+)-1,1'-Bi-2-naphthol studied by time-resolved circular dichroism", J. Am. Chem. Soc. 130, 12783-12786 (2008).

[5] Simpson, J.E.; Daub, G.H.; Hayes, F.N., "The Synthesis of Some 2,2'-Dioxa-Bridged Biphenyls and 1,1'Binaphthyls", J. Org. Chem. 38, 1771 (1973).

[6] Niezborala, C.; Hache, F., "Measuring the dynamics of circular dichroism in a pump-probe experiment with a Babinet-Soleil compensator", J. Opt. Soc. Am. B 23, 2418-2424 (2006).

[7] Schmid, M., Martinez-Fernadez, L., Markovitsi, D., Santoro, F., Hache, F., Improta, R., Changenet, P., J. Phys. Chem. Lett. 10, 4089-4094 (2019). 